JMB

\title{
Crystal Structure of the Escherichia coli RNA Degradosome Component Enolase
}

\section{Karin Kühnel and Ben F. Luisi*}

Department of Biochemistry University of Cambridge, 80 Tennis Court Road, Cambridge CB2 1GA, UK

\begin{abstract}
The crystal structure of Escherichia coli enolase (EC 4.2.1.11, phosphopyruvate hydratase), which is a component of the RNA degradosome, has been determined at $2.5 \AA$. There are four molecules in the asymmetric unit of the $C 2$ cell, and in one of the molecules, flexible loops close onto the active site. This closure mimics the conformation of the substratebound intermediate. A comparison of the structure of the E. coli enolase with the eukaryotic enolase structures available (lobster and yeast) indicates a high degree of conservation of the hydrophobic core and the subunit interface of this homodimeric enzyme. The dimer interface is enriched in charged residues compared with other protein homodimers, which may explain our observations from analytical ultracentrifugation that dimerisation is affected by ionic strength. The putative role of enolase in the RNA degradosome is discussed; although it was not possible to ascribe a specific role to it, a structural role is possible.
\end{abstract}

(C) 2001 Academic Press

Keywords: enolase; degradosome; analytical ultracentrifugation;

dimer interface; active site to three subgroups according to their catalytic mechanism, and these are represented by the proteins mandelate racemase (EC 5.1.2.2), muconate cycloisomerase (EC 5.5.1.1) and enolase itself. ${ }^{4}$ The three subgroups share structural homology as well as functional similarity, and the active site in all these enzymes is situated at the $C$ termini of the $\beta$ strands of an $\beta / \alpha$ barrel.

The eight-stranded $\beta / \alpha$ barrel is a widely occurring protein architecture. ${ }^{5,6}$ It consists of a cylindrical $\beta$-sheet, built of eight $\beta$-strands, which are surrounded by eight $\alpha$-helices. The barrel domains of all members of the enolase superfamily have an unusual $\beta \beta \alpha \alpha(\beta \alpha)_{6}$ topology ${ }^{1}$ differing from the more common $(\beta \alpha)_{8}$ topology of the barrels represented by triose-phosphate isomerase (TIM) (EC 5.3.1.1). ${ }^{7}$ In addition to the barrel domain, enolase also contains an $N$-terminal $\alpha / \beta$-domain consisting of a three-stranded antiparallel $\beta$-sheet and four $\alpha$ helices (Figure 1).

Recently enolase has been identified as a component of the RNA degradosome complex isolated from E. coli. 8 ,9 The degradosome consists of three other principal components in addition to enolase: ribonuclease E, polynucleotide phosphorylase (PNPase; EC 2.7.7.8 polyribonucleotide nucleotidyltransferase), and the RNA helicase RhlB. Ribonuclease $\mathrm{E}$ forms the scaffold of the degradosome, and the other three components of the complex bind to its C-terminal half. The RNase E binding 


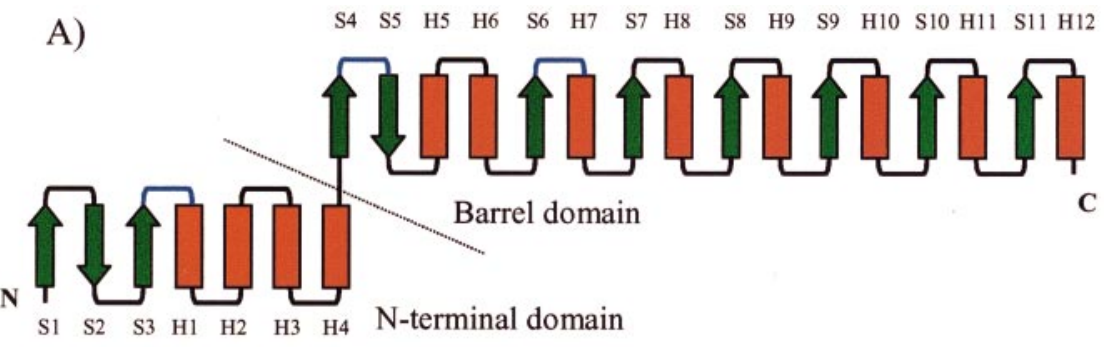

B)

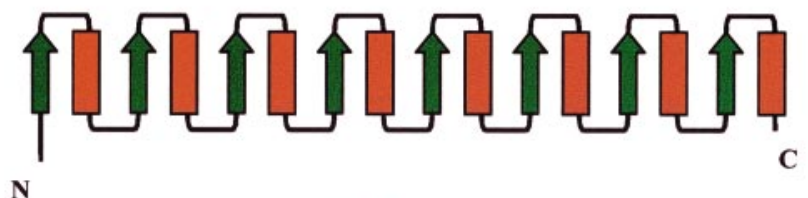

TIM barrel
Figure 1. Topology of (a) enolase and (b) the triosephosphate isomerase (TIM) barrel. The labelling convention used here for the strands and helices is shown. Because the enolase barrel domain has the unusual $\beta \beta \alpha \alpha(\beta \alpha)_{6}$ topology, S5 is antiparallel to the other strands of the barrel domain and $\mathrm{H} 5$ is antiparallel to the other barrel helices. Loops that change conformation upon binding of the substrate are labeled blue (between S4 and S5, S6 and $\mathrm{H7}$, and S3 and H1). The domain boundary for enolase is indicated. site for enolase comprises residues $739-845 .^{10}$ It appears that only a small portion of the total cellular enolase is bound in the degradosome, estimated to be $5-10 \% .{ }^{8}$ Interestingly, cellular enolase copurifies with the over-expressed C-terminal half of RNase E, which indicates that the interaction of these two proteins can be independent of the other degradosome components (Anastasia Callaghan, unpublished results). There is no evidence of an interaction between enolase and PNPase or RhlB. ${ }^{10}$

It seems surprising that a glycolytic enzyme is a component of an RNA processing machine, and the function of enolase in this assembly is currently unknown. A minimal degradosome, assembled from all components except enolase, is capable of degrading RNA, indicating that enolase is not crucial for the complex's RNA degradation function in vitro. ${ }^{11}$ However, it is possible that enolase might play a subtle structural role in the complete assembly or it could serve to couple glycolytic and RNA degradative processes in vivo through allosteric effects.

Our laboratory has reported the crystal structure of PNPase from Streptomyces antibioticus, which is a close homologue of the E. coli enzyme. ${ }^{12}$ In our continuing efforts to characterise the degradosome components and sub-assemblies, we have also studied the E. coli enolase crystal structure, which we report here at $2.5 \AA$ resolution. We have found that both the open and closed conformations of the enzyme are present in the same crystal. A comparison of the eukaryotic and prokaryotic enolase structures reveals that the dimer interfaces and hydrophobic core are well conserved. The dimer interface is enriched with charged residues in comparison with other homodimeric proteins, and analytical ultracentrifugation studies presented here suggest that electrostatic effects play an important role in stabilizing the homodimer.

One unexpected finding from the PNPase crystal structure was a structural similarity of a helical domain from this ribonuclease with the N-terminal domain of enolase. ${ }^{12}$ It is not clear, however, whether the structural similarity indicates a role for this domain in RNA binding. Yeast and E. coli enolase have been shown to bind RNA weakly in earlier studies ${ }^{13}$ (C. Burns and Y.-S. Li, personal communication), and this feature might be related to enolase's function in the degradosome. We attempted to co-crystallize E. coli enolase with $\mathrm{rU}_{8}$ and to soak 8-bromoadenosine $5^{\prime}$-diphosphate into enolase crystals, but we found no evidence of either potential ligand bound to the protein.

\section{Results}

\section{Architectural features}

The structure of $E$. coli enolase has been refined to $2.5 \AA$ resolution (Table 1). The enzyme is kidney shaped with a deep cavity in the barrel domain leading to the active site, which can be seen in the $\mathrm{C}^{\alpha}$-trace of the molecule shown in Figure 2. There are two dimers in the asymmetric unit of the crystal. Three of the subunits (A, B, C) are in the open conformation with the active site fully accessible. However, the fourth subunit (D) is in the closed conformation and its active site is occluded. Here, a loop from the $\mathrm{N}$-terminal domain, connecting the third strand and first helix, residues 38-46, closes on the active site moving approximately $11 \AA$ and another loop, residues 154-165, moves $3 \AA$ closer to the active site. Also the loop comprising residues 248-268 moves by roughly 4 A. A superimposition of the open and closed conformations is shown in Figure 3 . The closed conformation mimics the conformation observed in yeast enolase in complex with substrates and inhibitors. ${ }^{2,14-18}$ However, the E. coli enolase structure represents the first occurrence of the closed conformation in the absence of substrates and inhibitors.

There is no evidence that the enolase subunits work in a correlated manner and the asymmetry in the crystal is unlikely to arise from allostery. 
Table 1. Refinement statistics

\begin{tabular}{|c|c|}
\hline Protein atoms (residues) present in the structure & $12,340(1716)$ \\
\hline Solvent atoms (oxygen atoms) & 509 \\
\hline Magnesium ions & 5 \\
\hline Protein atoms (residues) missing from the structure & $361(8)$ \\
\hline Resolution limits ( $($ ) (last shell: $2.47-2.56 \AA$ ) & 28.0-2.47 \\
\hline Total reflections (test-set for $R_{\text {free }}$ ) & $63,664(6458)$ \\
\hline Completeness (\%) (last shell) & $93.8(72.7)$ \\
\hline$R_{\text {merge }}(\%)$ (last shell) & $5.3(10.7)$ \\
\hline$R$-factor (\%) (last shell) & $22.9(26.0)$ \\
\hline$R_{\text {free }}(\%)$ (last shell) & $27.6(31.8)$ \\
\hline Overall $B$-factor $\left(\AA^{2}\right)$ & 49.1 \\
\hline r.m.s.d. of subunit A to B $(\AA)$ & 0.33 \\
\hline r.m.s.d. of subunit A to $C(\AA)$ & 0.37 \\
\hline r.m.s.d. of subunit A to $\mathrm{D}(\AA)$ & 0.95 \\
\hline \multicolumn{2}{|l|}{ Stereochemistry } \\
\hline r.m.s.d. in bond lengths $(\AA)$ & 0.007 \\
\hline r.m.s.d. in bond angles (deg.) & 1.1 \\
\hline
\end{tabular}

Instead the crystal lattice contacts must be selectively stabilising the closed conformation of subunit D. The crystal structure has been reported of an asymmetric dimer of yeast enolase that was prepared from an equilibrium mixture of G2P and phosphoenolpyruvate. ${ }^{18}$ One subunit was present in the closed conformation, while the second was observed in a "loose" intermediate conformation.

The E. coli, yeast, and lobster structures are very similar. The three proteins differ only slightly in the conformation of loops, as shown in Figure 4. The largest difference between the prokaryotic and eukaryotic enolases is in the loop comprising residues 248-268. E. coli enolase has a corresponding five residue deletion between residues 259 and 260 . Compared to animal enolases, this loop is on average four residues shorter in prokaryotic enolases and two residues longer in plant enolases. ${ }^{18}$ This loop moves during the closure of the active site upon substrate binding. The root-mean-square fit of $\mathrm{C}^{\alpha}$ atom positions for the core regions, excluding loops, is $0.71 \AA$ and $0.76 \AA$ for matching the E. coli enzyme with yeast (3enl) and lobster (1pdy) eno- lases, respectively. For comparison, the fit of the corresponding portions of yeast and lobster enolases is $0.49 \mathrm{~A}$.

Enolase consists of two domains (Figures 1 and 2). The smaller N-terminal domain (residues 1-142) is a three-stranded anti-parallel $\beta$-sheet with tight turns, which is followed by four $\alpha$-helices. The strands S1, S2 and the loop connecting S3 and helix H1, which closes onto the active site upon substrate binding, are part of the dimer interface. Residues Gly72 and Pro73 introduce a bend into H1 (Figure 2). This kink is also present in the yeast and lobster enolase structures, but it occurs one turn later compared with the E. coli enolase.

Intriguingly, an $\alpha$-helical domain of PNPase from Streptomyces antibioticus, is similar to the first three $\alpha$-helices of the $\mathrm{N}$-terminal domain of enolase. $^{12}$ The $\alpha$-helical domain of PNPase also contains a helix with a bend induced by a proline residue, as shown in Figure 5. Perhaps the helical domain is involved in RNA binding for both these degradosome proteins, but this hypothesis awaits testing. PNPase contains KH and S1 RNA-binding
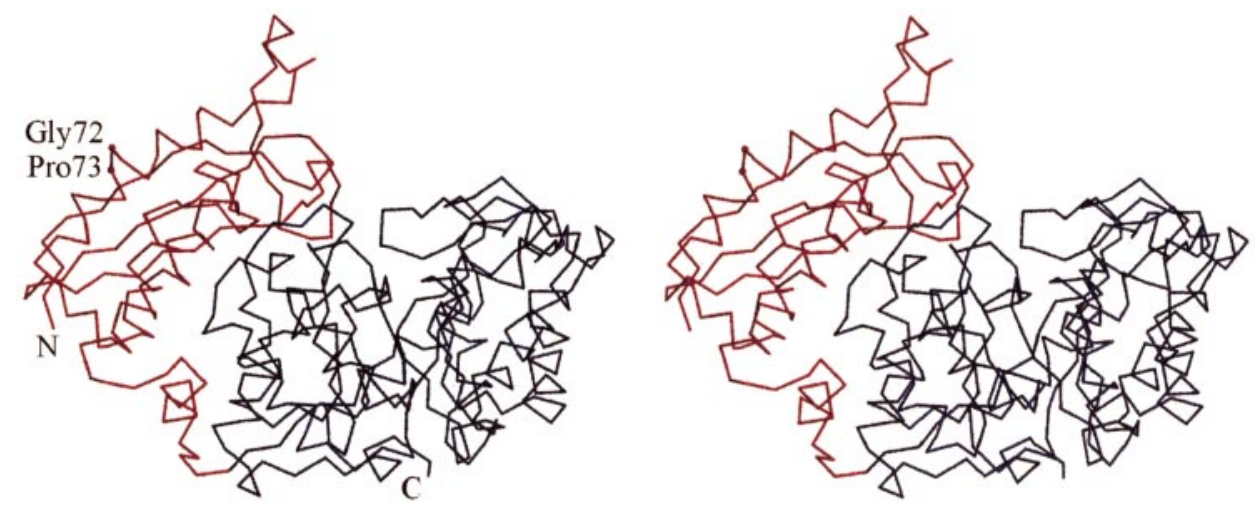

Figure 2. Stereo view of E. coli enolase $C^{\alpha}$-trace. The $\mathrm{N}$-terminal domain is shown in red and the barrel domain in blue. Residues Gly72 and Pro73, which introduce a kink into the first helix of the N-terminal domain, are labeled. The Figure was prepared with Molscript. ${ }^{42}$ 

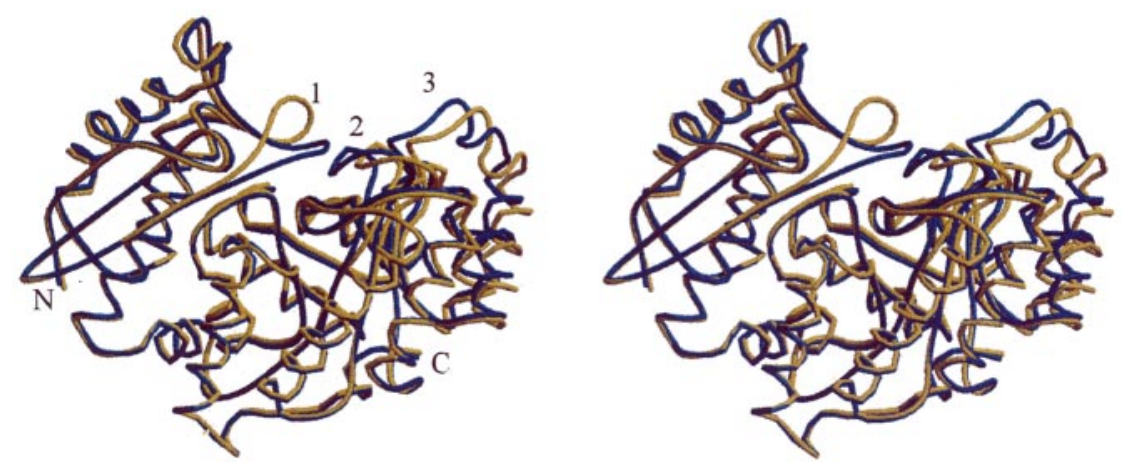

Figure 3. Subunits $A$ and D superimposed. In the closed conformation (subunit D) (1) loops 38-46, (2) 154-165 and (3) 248-268 move towards the active site.

domains, and the helical domain of PNPase might represent an additional, independent binding site.

Like other members of the TIM barrel architectural family, the enolase principal domain (residues 143-431) is an 8-fold barrel. However it deviates from the simpler $(\beta \alpha)_{8}$ topology of the TIM barrel and has an unusual $\beta \beta \alpha \alpha(\beta \alpha)_{6}$ organisation (Figure 1). With the distinctive topology of the enolase barrel, S5 is antiparallel to the other barrel strands and the first helix of the barrel H5 is antiparallel to the other barrel helices. H5 is also the longest helix (residues 178-198) of the protein. It forms an extended entrance to the active site and contributes to the dimer interface. The helices of the barrel are amphiphilic in character. The exception is the mainly hydrophobic helix H11 (378-385) of the barrel, which is at the interface between the barrel and the $\mathrm{N}$-terminal domain. The $\beta$-strands of the barrel are three to five residues long. Usually the first amino acid residues are hydrophobic and the last one, which participates in the active site, is hydrophilic.

Ala335 is in the loop connecting H9 and S9 of the barrel, and in all four molecules in the asym-

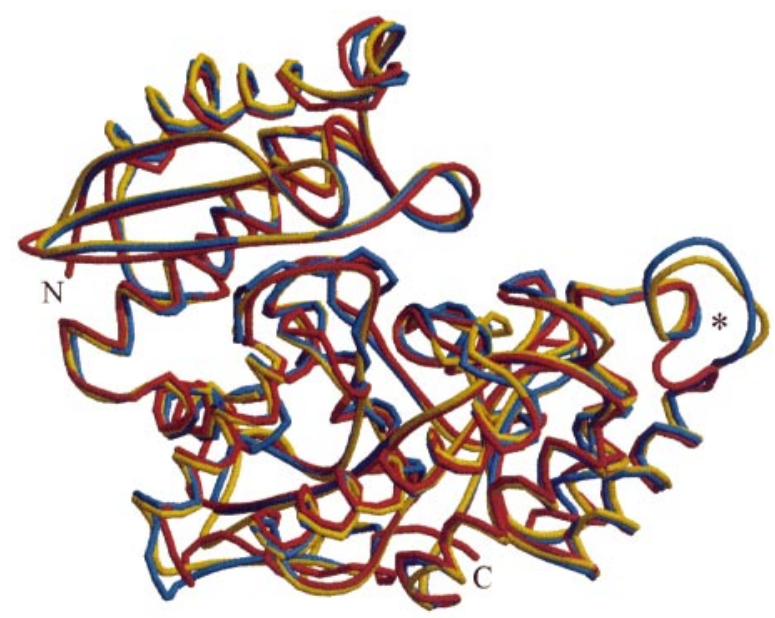

Figure 4. Comparison of E. coli (red), yeast (yellow; 3enl) and lobster (blue; 1pdy) enolase structures. An asterisk marks loop 250-267, which contains a five residue deletion in E. coli. Superimposition was done with Comparer. ${ }^{43}$ metric unit, this residue is in the generously allowed portion of the Ramachandran plot. Its distinctive geometry is stabilized through hydrogen bonds between the amide nitrogen atom of Ala335 and the hydroxy group of Thr304, and by a second hydrogen bond between the carbonyl oxygen atom Ile334 and the hydroxy group of Tyr334.

\section{The active site}

The active site is situated at the C-terminal ends of the $\beta$-strands of the barrel, similar to other enzymes with an 8-fold barrel domain. The active sites of E. coli, yeast, and lobster enolase are very similar structurally, and all the residues involved in catalysis are conserved. As Grishin \& Phillips have noted, active site residues are generally the most conserved residues in proteins, evolving 50 times slower on average than other residues. ${ }^{19}$ The active site residues of E. coli enolase superimpose very closely with the corresponding yeast and lobster residues in the open and closed conformation.

Enolase catalyses the dehydration of 2-phosphoglycerate in a stepwise manner ${ }^{20,21}$ through an anti $\beta$-elimination mechanism. ${ }^{22}$ Initially, a base abstracts the proton from C2 of 2-phosphoglycerate, forming a carbanion. In the second step of catalysis the hydroxy group from C3 is eliminated by general-acid catalysis. Lys345 (E. coli: Lys341) and Glu211 (E. coli: Glu208) have been proposed to act as a catalytic base-acid pair. ${ }^{17,23}$ An alternative proposal suggests that Glu211 and Glu168 share a proton, which then catalyzes the abstraction of the hydroxy group. ${ }^{18}$

In yeast and lobster enolase, it has been observed that the binding of substrate causes major conformational changes in the enzyme that closes the substrate into the active site. In yeast enolase, the $\mathrm{N}$-terminal domain contributes a long flexible loop that closes on the active site by binding a magnesium ion via residue Ser39 (corresponding to E. coli residue 41) and a second loop (153-169) containing His159 (E. coli: His158) moves closer to the active site. ${ }^{2,14-18}$ Two magnesium ions per subunit are required for enolase's catalytic activity. ${ }^{24}$ The second magnesium ion has lower affinity and binds after the substrate has bound..$^{25,26}$ 


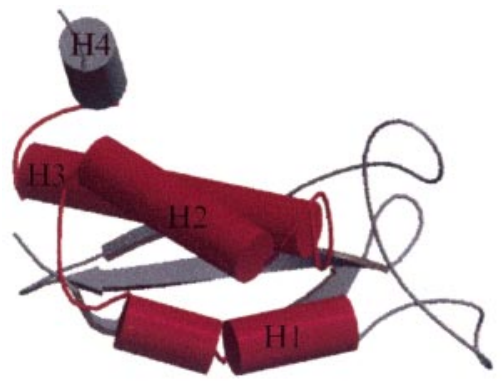

A)

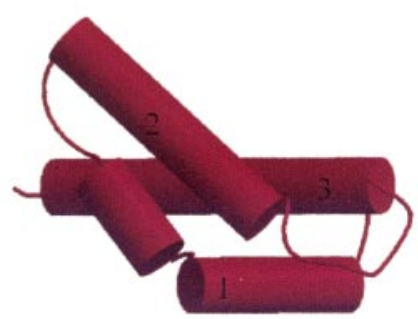

B)
Figure 5. Similarity between the first three helices (red) of (a) the Nterminal domain of enolase and (b) the helical domain of polynucleotide phosphorylase.
In the E. coli enolase structure, one of the active site magnesium ions is bound by Asp245, Glu289 and Asp316. In the closed conformation of subunit $\mathrm{D}$, electron density was also noted for a potential second magnesium ion, and the metal was included in the model. This density is in the same position as the second magnesium ion found in the yeast enolase complex with G2P and phosphoenolpyruvate $^{17}$ and with an intermediate analogue. $^{2}$ Also at the closed-state D subunit of the E. coli enzyme, electron density was observed at the position corresponding to the substrate's phospho group in the yeast enolase complex (Figure 6). We placed a sulfate ion here, because the height of the peak was comparable to the electron density of the sulfur atoms of the methionine and cysteine side-chains. In the substrate-free forms of both yeast and lobster enolase, a sulfate ion was found at the corresponding position.,27 We note that both the yeast and lobster free enolases were crystallised from high concentrations of ammonium sulfate. There was no sulfate in the crystallisation buffer of the E. coli enzyme; however, the purification procedure involved precipitation and hydrophobic chromatography with ammonium sulfate, and it seems likely that the enzyme acquired the sulfate ion at this stage. Curiously, there is no electron density that could be attributed to a sulfate ion in the open active sites of the A,B or C subunits in the E. coli crystal. This suggests that binding of the sulfate requires the second magnesium ion and the closed conformation in the E. coli enzyme.

\section{The dimer interface}

The E. coli enolase dimer is shown in Figure 7. Like the core, the dimer interface is also structurally conserved. The dimer interfaces of E. coli, yeast, and lobster enolase have similar amounts of buried surface area and similar surface contact shapes and charge distributions (Figure 8). The conservation of the dimer interface between the eukaryotic and prokaryotic enolases is intriguing. The enzyme has no known cooperative effects, so the interface does not serve any allosteric function.

$\dagger$ Available online at www.biochem.ucl.ac.uk/bsm/ $\mathrm{PP} /$ server/
Indeed, yeast enolase monomers, which dissociated at $40^{\circ} \mathrm{C}$, were shown to be active in the presence of $\mathrm{Mg}^{2+}$, substrate, and product. ${ }^{28}$ Perhaps dimerisation promotes the stability of the subunits. The E. coli enolase subunit contacts in the dimer bury $3320 \AA^{2}$, which is similar to the average buried surface of $3370 \AA^{2}$ found for other homodimeric proteins. ${ }^{29}$ The dimer interface of lobster enolase buries a similar surface area of $3740 \AA^{2} .^{3}$

Further analysis of the E. coli enolase dimer interface with the "Protein-Protein Interaction Server ${ }^{\prime 29} \uparrow$ shows that the enolase dimer interface is more planar than the average homodimer interface. The value for the interface planarity, defined as the r.m.s.d. of the interface atoms from the bestfit plane through the interface, in the E. coli enolase dimer is $1.7 \AA$ as compared to an average value of

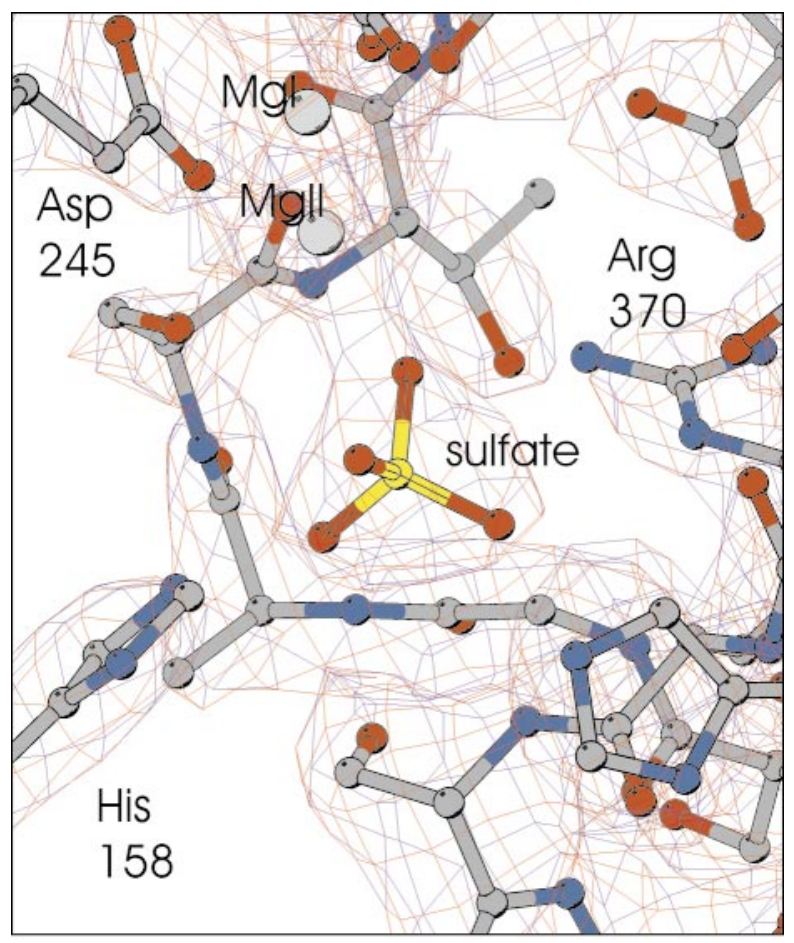

Figure 6. Electron density at the active site of the closed state (subunit D), showing the proposed sulfate ion and the two magnesium ions. The map was calculated using minimal bias coefficients. 


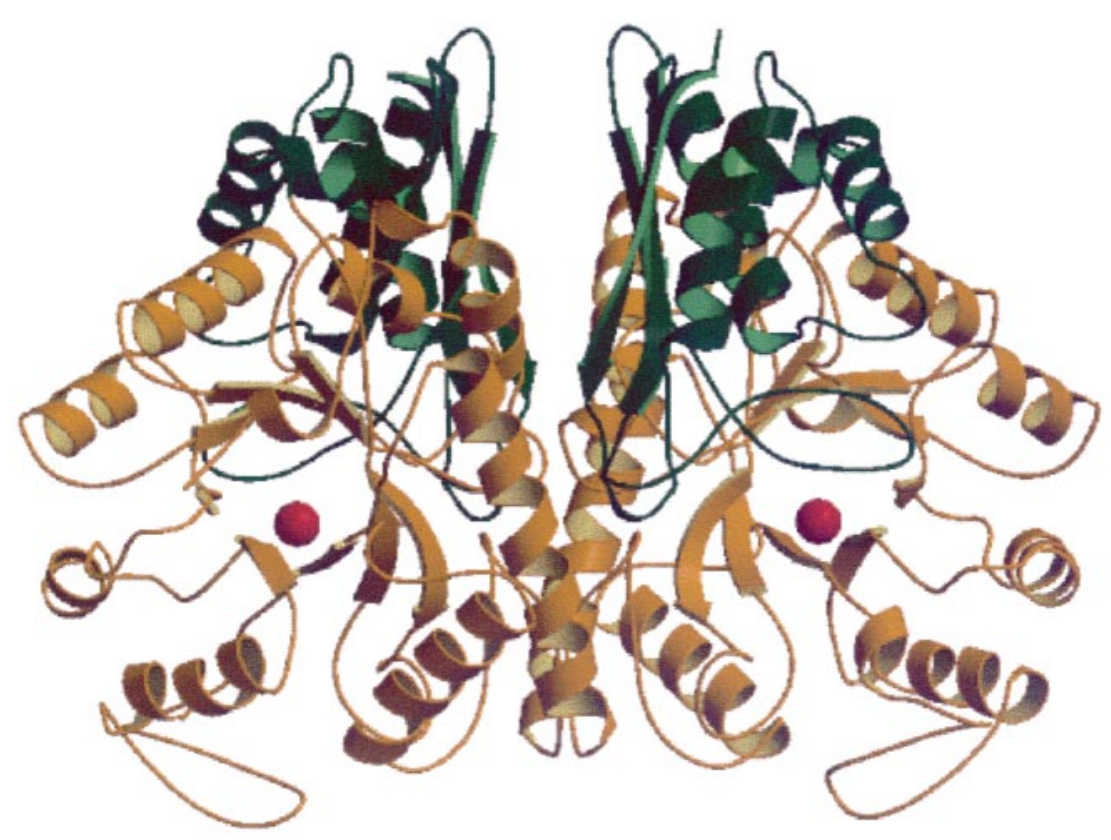

Figure 7. The E. coli enolase dimer. The dyad axis is oriented vertically. The N-terminal domain is green, the barrel domain is gold and the $\mathrm{Mg}$ ions are red spheres.

$3.5 \AA$. The gap volume index, ${ }^{29}$ defined as the ratio of void volume between molecules and interface accessible surface area, gives a measure of the complementarity of the interacting surfaces. The gap volume index for enolase is 3.2 , which is greater than the average value for homodimers of $2.2 \pm 0.9$, showing that the enolase complex is less complementary.

The ratio of charged/hydrophobic interface residues is 1.5 for enolase, more than twice the average of 0.7 for oligomeric proteins, ${ }^{30}$ because the enolase dimer interface is more polar than most other cases. The greater than expected polar/nonpolar ratio and the smaller than expected gap volume index may explain the observed salt dependency for dimer formation by E. coli enolase, which was studied by analytical ultracentrifugation. Enolase was dialyzed against water and different salts were added to a concentration of $5 \mathrm{mM}$. The average molecular mass was determined for every sample, from which the monomer/dimer ratio could be calculated (see Table 2). In pure water more than half of the molecules are dimeric. The proportion of dimers increases with ionic strength, and divalent cations seem to have a stronger effect in promoting dimerisation. The oligomerisation state in high ionic strength was also evaluated by small-angle X-ray scattering, and this confirms that enolase is a dimer in solution (Günter Grossmann and K.K., unpublished results).

Arg398 is part of the dimer interface and it lies in the loop connecting the last strand and helix of the barrel domain (S11 and H12). In all four subunits, Arg398 occupies the generously allowed lefthanded helical regions of the Ramachandran plot. The unusual conformation is stabilized through four intra- and intermolecular interactions. Firstly the carbonyl oxygen atom of Arg398 forms hydrogen bonds with the amide nitrogen atoms of Arg401 and Val402. Additionally there are two contacts across the dimer interface with the amide nitrogen atom of Arg398 forming a hydrogen bond with the carboxy oxygen atom of Ser14, and the amide nitrogen atom of Ser399 interacts with the carboxy group of the Asp400 in the other subunit. Interestingly, the residues corresponding to Arg398 in yeast enolase (Arg402 (1one, 2one)) and lobster

Table 2. Salt dependency of dimerisation from AUC

\begin{tabular}{|c|c|c|c|}
\hline Solution ( $25 \mu \mathrm{M}$ enolase) & Average $M_{\mathrm{r}}$ & \% Monomer & \% Dimer \\
\hline Water & 73,000 & 41 & 59 \\
\hline $5 \mathrm{mM} \mathrm{MgCl}_{2}$ & 92,000 & 0 & 100 \\
\hline $5 \mathrm{mM} \mathrm{Mg}(\mathrm{OAc})_{2}$ & 93,000 & 0 & 100 \\
\hline $5 \mathrm{mM} \mathrm{NaCl}$ & 85,000 & 15 & 85 \\
\hline $5 \mathrm{mM} \mathrm{CaCl}_{2}$ & 91,000 & 2 & 98 \\
\hline $5 \mathrm{mM} \mathrm{Na}_{2} \mathrm{SO}_{4}$ & 86,000 & 13 & 87 \\
\hline $5 \mathrm{mM}\left(\mathrm{NH}_{4}\right)_{2} \mathrm{SO}_{4}$ & 85,000 & 15 & 85 \\
\hline
\end{tabular}


A)

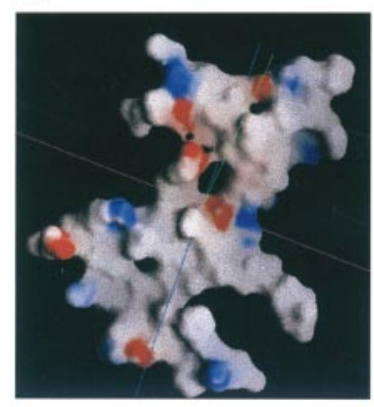

B)

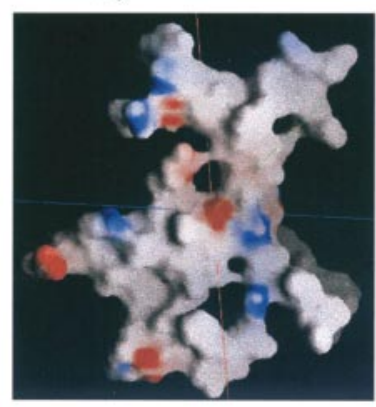

C)

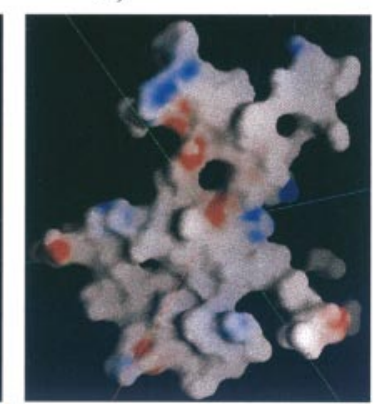

Figure 8. Electrostatic surface potential of enolase dimer interfaces. (a) E. coli enolase. (b) Yeast enolase (1ebh). (c) Lobster enolase $(1 \mathrm{pdz})$. Only other subunit-contacting residues are shown. A similar perspective was chosen for all three interfaces. Negative potential is indicated by red coloring and positive is labeled blue. Histidine residues were assumed to be neutral. The Figure was prepared with GRASP. ${ }^{44}$

enolase (Arg401 (1pdy)) are also in the generously allowed region, which underscores the detailed conservation of the dimer interface.

\section{Evaluation of potential RNA and nucleotide binding}

Yeast enolase binds single and double-stranded DNA and RNA, and upon binding of nucleic acid an inhibition of enolase activity was observed. ${ }^{13}$ This suggests that the conformation of the active site might somehow influence the binding of nucleic acid. Recently, it has also been found that E. coli enolase binds RNA (C.Burns and Y-S. Li, personal communication). In light of these observations, we attempted to co-crystallize enolase and an $\mathrm{rU}_{8}$ oligonucleotide. We could grow enolase crystals in the presence of RNA under conditions similar to those used to crystallize the native protein alone, and these crystals were of a different form and space group $\left(P 4_{2}\right.$ versus $\left.C 2\right)$. The structure of the new crystal was solved by molecular replacement using the refined E. coli enolase structure from the $C 2$ crystal. The structure was refined with CNS carrying out rigid-body refinement, simulated annealing and individual $B$-factor refinement to an $R$-factor of $27.4 \% \quad\left(R_{\text {free }}=33.1 \%\right)$. Although the UV-spectra of washed and dissolved crystals indicated the presence of nucleic acid, no RNA was visible in the electron density. Attempts were also made to soak 8-bromoadenosine 5 -diphosphate into these crystals. The $R$-factor after refinement was $24.7 \%$ and the $R_{\text {free }} 30.6 \%$ for data up to $2.7 \AA$. There was no evidence for the nucleotide in the structure. In one of the two enolase molecules of the asymmetric unit, the enzyme is in the closed conformation and a phosphate ion was visible at the same position previously observed for the phosphate ion in the structure of yeast enolase complexed with fluoride and phosphate. ${ }^{15}$ Phosphate was not present in the crystallisation buffer but was very likely released from hydrolysis of 8-bromoadenosine $5^{\prime}$-diphosphate.

\section{Discussion}

The overall structure of E. coli enolase is very similar to the enolase structures of the eukaryotic organisms yeast and lobster, indicating that the enzyme has remained conserved at both the tertiary and quaternary structural levels throughout its evolution. Both the open and closed forms of E. coli enolase were captured in the crystal.

Other studies have suggested that enolase can bind DNA and RNA. ${ }^{13}$ Based on these observations, attempts were made to co-crystallize enolase with RNA and to soak 8-bromoadenosine 5 -diphosphate into the crystal. No electron density for the RNA or the nucleotide was visible. Instead, the active site was found to be in the closed conformation with possibly a phosphate ion bound in the case of the 8-bromoadenosine 5'-diphosphate soak. The position of this putative phosphate position corresponds to the phosphate group of the substrate 2-phosphoglycerate in the substrate/enzyme complex. At the same position weaker electron density was visible in the enolase $/ \mathrm{rU}_{8}$ crystals, where a putative sulfate ion may be bound with partial occupancy.

The role of enolase in the degradosome is still unknown. It might have a structural role or couple RNA degradation to glycolysis. Other RNAdegrading complexes, including the exosome, ${ }^{31,32}$ have been identified in eukaryotic organisms and are not known to contain enolase. Also the observation that a functional minimal degradosome consisting of RNase E, PNPase and RhlB could be assembled in vitro ${ }^{11,33}$ does not support the idea of a crucial role of enolase in RNA degradation. It seems more likely that enolase may play a structural role in the E. coli degradosome, and perhaps an allosteric role to affect the activity of its linked partners. We have observed that a fragment encompassing residues 628 to 843 from RNase E does not support a complex with enolase, while it interacts avidly with the degradosome RNA helicase and much more weakly with PNPase (results not shown). Also, enolase does not interact directly with PNPase, helicase, or the complex of helicase 
with the above peptide at micromolar concentrations. However, if the components are in close physical proximity through binding of a common peptide, even a weak intermolecular interaction would be favoured and the enzymes could mutually influence the activities of their neighbours. The role of enolase in the degradosome and its contribution to the assembly might be better understood by structurally characterizing its interactions with peptides from the C-terminal half of RNase $\mathrm{E}$ that encompass its binding site.

\section{Experimental Procedures}

\section{Purification of enolase}

The E. coli enolase gene was cloned into the pET11a vector (Novagen) by Dr A.J. Carpousis (Toulouse) and transformed into BL21(DE3) E. coli cells (Novagen). Cultures were grown at $37^{\circ} \mathrm{C}$ in $\mathrm{LB}$ medium containing $100 \mathrm{mg} / 1$ ampicillin to an absorbance of $0.5-0.6$ at $600 \mathrm{~nm}$, then induced with $1 \mathrm{mM}$ IPTG and incubated for three hours at $37^{\circ} \mathrm{C}$. Bacteria were harvested by centrifugation at $7000 \mathrm{~g}$ for ten minutes. Pellets from a 11 culture were resuspended in $30 \mathrm{ml}$ of $20 \mathrm{mM}$ Tris (pH 8.0). Cells were lysed with a French Press (SLM Instruments). Cell debris was removed by centrifugation at $25,000 \mathrm{~g}$ for 20 minutes at $4{ }^{\circ} \mathrm{C}$, and the supernatant was then diluted to $50 \mathrm{ml}$ with pre-cooled $20 \mathrm{mM}$ Tris ( $\mathrm{pH}$ 8.0). Ammonium sulfate was added to $65 \%$ saturation $(398 \mathrm{~g} / \mathrm{l})$. The solution was stirred for one hour at $4{ }^{\circ} \mathrm{C}$ and then spun at $30,000 \mathrm{~g}$ for 30 minutes at $4{ }^{\circ} \mathrm{C}$. Ammonium sulfate was added to the supernatant to $95 \%$ saturation $(205 \mathrm{~g} / \mathrm{l})$ to precipitate enolase. After stirring at $4{ }^{\circ} \mathrm{C}$ for one hour, the sample was centrifuged at $30,000 \mathrm{~g}$ for 30 minutes at $4{ }^{\circ} \mathrm{C}$. The $95 \%\left(\mathrm{NH}_{4}\right)_{2} \mathrm{SO}_{4}$ pellet from a 11 preparation was resuspended in a $50 \mathrm{ml}$ of buffer A (1.9 M ( $\left.\mathrm{NH}_{4}\right)_{2} \mathrm{SO}_{4}, 5 \mathrm{mM} \mathrm{MgCl}, 20 \mathrm{mM}$ Tris $(\mathrm{pH} 8.0))$ and loaded onto a Phenyl Sepharose High Performance HiLoad 16/10 column (Amersham-Pharmacia). A gradient was applied to the column from $0 \%$ to $100 \%$ buffer B (5 mM $\mathrm{MgCl}_{2}, 20 \mathrm{mM}$ Tris (pH 8.0)) over $200 \mathrm{ml}$. Fractions enriched in enolase were pooled and enolase was precipitated by adding $470 \mathrm{~g} / \mathrm{l}\left(\mathrm{NH}_{4}\right)_{2} \mathrm{SO}_{4}$ to a final concentration of $95 \%$. The sample was stirred at $4{ }^{\circ} \mathrm{C}$ for one hour and spun at $30,000 \mathrm{~g}$ for 30 minutes. The pellet was resuspended in $4 \mathrm{ml}$ of buffer $\mathrm{C}(150 \mathrm{mM}$ $\mathrm{NaCl}, 20 \mathrm{mM} \mathrm{MgCl} 2,20 \mathrm{mM}$ Tris ( $\mathrm{pH}$ 8.0)), dialyzed against 11 of buffer $\mathrm{C}$ for one hour at $4{ }^{\circ} \mathrm{C}$ and concentrated to $2 \mathrm{ml}$. Samples $(500 \mu \mathrm{l})$ were loaded onto a Superdex200 HR10/30 column (Amersham-Pharmacia) and eluted with $25 \mathrm{ml}$ of buffer C. Fractions were stored at $-20^{\circ} \mathrm{C}$. The molecular mass of enolase was measured with a BioQ mass spectrometer and data were analyzed with Mass Lynx software giving a value of $45,545( \pm 35)$ $\mathrm{Da}$, which is close to the expected value of 45,525 . The protein concentration was determined by measuring the absorbance at $280 \mathrm{~nm}$ with a UV-1201 spectrophotometer (Shimadzu). The extinction coefficient of enolase $\left(19770 \mathrm{M}^{-1} \mathrm{~cm}^{-1}\right)$ was calculated with ProtParam. ${ }^{34}$

\section{Enolase assay}

Enolase catalyzes the dehydration of G2P to phosphoenolpyruvate, and the formation of the product was followed at $240 \mathrm{~nm}$. Assays were carried out at room temperature in medium containing $1 \mathrm{mM}$ 2-phosphogly- cerate, $1 \mathrm{mM} \mathrm{MgSO}_{4}, 100 \mathrm{mM} \mathrm{KCl}, 50 \mathrm{mM}$ Tris (pH 8.1), and $0.01 \mathrm{mM}$ EDTA. ${ }^{35} 5 \mu \mathrm{g}$ of enolase was added to $1 \mathrm{ml}$ of the assay medium to start the reaction. The activity of the purified enolase was found to be $260( \pm 13)$ $\mathrm{U} / \mathrm{mg}$, where $1 \mathrm{U}=1 \mu \mathrm{mol} / \mathrm{min}$.

\section{Analytical ultracentrifugation (AUC)}

Equilibrium sedimentation experiments were carried out with a Beckman Optima XL-I ultracentrifuge. Purified enolase was dialyzed extensively against water, then diluted to $25 \mu \mathrm{M}$ and different salts were added to a final concentration of $5 \mathrm{mM}$. The samples were centrifuged first at $12,000 \mathrm{rpm}$, then at $15,000 \mathrm{rpm}$ and finally at 18,000 rpm; each step was for 24 hours and the temperature was maintained at $5^{\circ} \mathrm{C}$ throughout the procedure. Data were analyzed with the Beckman/Origin Optima $^{\mathrm{TM}}$ XL-A/XL-I Data Analysis Software v4. The average molecular mass was determined for each sample. From the average molecular mass, the monomer/dimer ratio could be calculated.

\section{Crystallization and data collection}

Purified enolase was dialyzed against $40 \mathrm{mM} \mathrm{NaCl}$, $5 \mathrm{mM} \mathrm{MgCl}$ at $4{ }^{\circ} \mathrm{C}$ and concentrated. Crystals were grown by vapour diffusion at $20^{\circ} \mathrm{C}$ using the sitting drop method. Ten microlitres of $70 \mathrm{mg} / \mathrm{ml}$ enolase were mixed with $10 \mu \mathrm{l}$ of $20 \%$ (w/v) PEG3350, $200 \mathrm{mM}$ magnesium acetate and equilibrated against 20\% PEG3350, $200 \mathrm{mM}$ magnesium acetate. Elongated birefringent crystals grew to a size of $1000 \mu \mathrm{m} \times 250 \mu \mathrm{m} \times 250 \mu \mathrm{m}$ within two weeks. Crystals were frozen in the mother liquor supplemented with $20 \%$ (w/v) PEG400. Data were collected at $100 \mathrm{~K}$ on Elettra beamline 5.2R, Trieste, Italy, at $\lambda=1.472 \AA$ using a MAR345 detector. The data were processed with DENZO and SCALEPACK. ${ }^{36}$ Data were $97.6 \%$ complete to $2.47 \AA$, with an average multiplicity of observation of 4.6 and an average intensity of 10.4 standard deviations in the $2.56-2.47 \AA$ resolution shell. The overall $R_{\text {merge }}$ was $5.3 \%$. The crystals belong to space group C2 with unit cell dimensions $a=107.9 \AA$, $b=150.0 \AA, c=127.4 \AA$ and $\beta=109.2^{\circ}$. Calculation of the Matthews coefficients indicated the presence of four molecules in the asymmetric unit $\left(V_{\mathrm{M}}=2.7 \AA^{3} / \mathrm{Da}\right)$.

\section{Co-crystallization enolase/RNA}

Enolase $\left(45 \mathrm{mg} / \mathrm{ml}\right.$ in $\left.40 \mathrm{mM} \mathrm{NaCl}, 5 \mathrm{mM} \mathrm{MgCl}_{2}\right)$ was mixed in a 1:1.5 ratio with HPLC-purified $\mathrm{rU}_{8}$ (Oswel) dissolved in $40 \mathrm{mM} \mathrm{NaCl}, 5 \mathrm{mM} \mathrm{MgCl}$. Spermine tetrahydrochloride $(1 \mathrm{mM})$ was added. Protein/ RNA $(2 \mu \mathrm{l})$ and $2 \mu \mathrm{l}$ of the reservoir solution ( $0.2 \mathrm{M}$ magnesium formate, $20 \%$ PEG3350) were mixed and equilibrated against the reservoir solution at $20^{\circ} \mathrm{C}$. Long needles grew after one week. The crystals diffracted to $2.5 \AA$ using a Rigaku/R-AXIS IV generator and detector. The quotient of the average intensity by the standard deviation was 4.2 for the $2.59-2.50 \AA$ shell, and the overall $R_{\text {merge }}$ was $4.3 \%$. Crystals are tetragonal (space group $P 4_{2}$ ) with $a=b=124.1 \AA$ and $c=55.8 \AA$.

Crystals were also soaked with nucleotide. The crystals grown in the presence of $\mathrm{rU}_{8}$ were transferred for four minutes into the cryoprotectant $(1 \mathrm{mM}$ magnesium formate, 35\% PEG600) containing $2 \mathrm{mM}$ 8-bromoadenosine 5'-diphosphate (Sigma) and then frozen. Data were collected to $2.7 \AA$ using a Rigaku/R-AXIS IV generator and detector, with an average $I / \sigma$ of 3.5 in the $2.8-2.7 \AA$ 
shell and overall $R_{\text {merge }}$ of $6.9 \%$. The $\mathrm{P} 4_{2}$ crystals had the cell dimensions $a=b=124.6 \AA, c=56.2 \AA$.

\section{Structure determination}

The structure of the native enolase was solved by Molecular Replacement with $\mathrm{AMoRe}^{37,38}$ using a yeast enolase dimer as a search model (1ebg). ${ }^{2}$ Two dimers were placed into the asymmetric unit. Strict non-crystallographic symmetry (NCS) was applied and iterative cycles of rigid body refinement, simulated annealing, individual $B$-factor refinement with $\mathrm{CNS}^{39}$ and 4-fold non-crystallographic symmetry averaging and solvent flattening with $\mathrm{DM}^{40}$ were carried out. QUANTA (Molecular Simulations Inc., 1997) was used for inspection of the electron density maps, models and the manual rebuilding of the model structure. After the first two cycles of refinement the yeast residues were mutated to the corresponding E. coli residues. Then cycles of simulated annealing, energy minimisation and $B$-factor refinement with CNS and model rebuilding were repeated with strict NCS. Later NCS was restrained and it became clear from the difference map $\left|F_{\mathrm{o}}\right|-\left|F_{\mathrm{c}}\right|$ that loops in one of the subunits (referred to throughout as subunit D) comprising residues 38-46 and 154-165 differed significantly from the other three subunits in the asymmetric unit and needed to be rebuilt. In the final stages of refinement the NCS restraint was relaxed and $\mathrm{Mg}^{2}+$ was built into the active site. Water molecules were placed automatically with CNS and manually with QUANTA at positive peaks of the difference map $\left|F_{\mathrm{o}}\right|-\left|F_{\mathrm{c}}\right|$. In total, 509 water molecules were introduced into the model. The final $R$-factor is $22.9 \%$, the $R_{\text {free }} 27.6 \%$. Subunits A and D are in good density, with only a few flexible side-chains disordered at the surface. The electron densities of subunits $B$ and $C$ are decidedly poorer, due to the greater conformational variations of these subunits. Approximately $10 \%$ of the residues of subunits B and $C$ were modeled as alanine because of poor electron density for the side-chains. A summary of the refinement statistics is presented in Table 1 .

Structure validation was done with PROCHECK: ${ }^{41}$ $85.5 \%$ of residues are in the core region of the Ramachandran plot, $14.0 \%$ in the allowed regions, $0.5 \%$ in the generously allowed regions and no residue is in the disallowed region.

\section{Protein Data Bank accession numbers}

Coordinates for E. coli enolase have been deposited in the Protein Data Bank as entry 1e9i.

\section{Acknowledgements}

We thank our colleagues A.J. Carpousis (CNRS, Toulouse) for providing the expression construct of E. coli enolase, Matthew Deacon for help with the analytical ultracentrifugation and Martyn Symmons for help with the mass spectrometry analysis. For their excellent advice and invaluable discussions, we thank A.J. Carpousis, Hal Dixon, Martyn Symmons, Kathryn Phillips, Frank Von Delft, Dima Chirgadze, Günter Grossmann and Anastasia Callaghan. This work was supported by the Wellcome Trust.

\section{References}

1. Lebioda, L., Stec, B. \& Brewer, J. M. (1989). The structure of yeast enolase at $2.25-\AA$ resolution. An 8 -fold beta + alpha-barrel with a novel beta beta alpha alpha (beta alpha)6 topology. J. Biol. Chem. 264, 3685-3693.

2. Wedekind, J. E., Poyner, R. R., Reed, G. H. \& Rayment, I. (1994). Chelation of serine 39 to $\mathrm{Mg}^{2+}$ latches a gate at the active site of enolase: structure of the bis $\left(\mathrm{Mg}^{2+}\right)$ complex of yeast enolase and the intermediate analog phosphonoacetohydroxamate at 2.1-Å resolution. Biochemistry, 33, 9333-9342.

3. Duquerroy, S., Camus, C. \& Janin, J. (1995). X-ray structure and catalytic mechanism of lobster enolase. Biochemistry, 34, 12513-12523.

4. Gulick, A. M., Hubbard, B. K., Gerlt, J. A. \& Rayment, I. (2000). Evolution of enzymatic activities in the enolase superfamily: crystallographic and mutagenesis studies of the reaction catalyzed by D-glucarate dehydratase from Escherichia coli. Biochemistry, 39, 4590-4602.

5. Murzin, A. G., Brenner, S. E., Hubbard, T. \& Chothia, C. (1995). SCOP: a structural classification of proteins database for the investigation of sequences and structures. J. Mol. Biol. 247, 536-540.

6. Hocker, B., Beismann-Driemeyer, S., Hettwer, S., Lustig, A. \& Sterner, R. (2001). Dissection of a (betaalpha)8-barrel enzyme into two folded halves. Nature Struct. Biol. 8, 32-36.

7. Banner, D. W., Bloomer, A. C., Petsko, G. A., Phillips, D. C., Pogson, C. I., Wilson, I. A. et al. (1975). Structure of chicken muscle triose phosphate isomerase determined crystallographically at 2.5 angstrom resolution using amino acid sequence data. Nature, 255, 609-614.

8. Py, B., Higgins, C. F., Krisch, H. M. \& Carpousis, A. J. (1996). A DEAD-box RNA helicase in the Escherichia coli RNA degradosome. Nature, 381, 169172.

9. Miczak, A., Kaberdin, V. R., Wei, C. L. \& Lin-Chao, S. (1996). Proteins associated with RNase E in a multicomponent ribonucleolytic complex. Proc. Natl Acad. Sci. USA, 93, 3865-3869.

10. Vanzo, N. F., Li, Y. S., Py, B., Blum, E., Higgins, C. F., Raynal, L. C. et al. (1998). Ribonuclease E organizes the protein interactions in the Escherichia coli RNA degradosome. Genes Dev. 12, 2770-2781.

11. Coburn, G. A., Miao, X., Briant, D. J. \& Mackie, G. A. (1999). Reconstitution of a minimal RNA degradosome demonstrates functional coordination between a $3^{\prime}$ exonuclease and a DEAD-box RNA helicase. (published erratum appears in Genes Dev. (2000);14:1167). Genes Dev. 13, 2594-2603.

12. Symmons, M. F., Jones, G. H. \& Luisi, B. F. (2000). A duplicated fold is the structural basis for polynucleotide phosphorylase catalytic activity, processivity, and regulation. Struct. Fold. Des. 8, 1215-1226.

13. al-Giery, A. G. \& Brewer, J. M. (1992). Characterization of the interaction of yeast enolase with polynucleotides. Biochim. Biophys. Acta, 1159, 134-140.

14. Lebioda, L. \& Stec, B. (1991). Mechanism of enolase: the crystal structure of enolase-Mg2(+)-2- phosphoglycerate/phosphoenolpyruvate complex at $2.2-\AA$ resolution. Biochemistry, 30, 2817-2822.

15. Lebioda, L., Zhang, E., Lewinski, K. \& Brewer, J. M. (1993). Fluoride inhibition of yeast enolase: crystal structure of the enolase- $\mathrm{Mg}(2+)-\mathrm{F}(-)-\mathrm{Pi}$ complex at 
$2.6 \AA$ resolution. Proteins: Struct. Funct. Genet. 16, 219-225.

16. Zhang, E., Hatada, M., Brewer, J. M. \& Lebioda, L. (1994). Catalytic metal ion binding in enolase: the crystal structure of an enolase- $\mathrm{Mn}^{2+}$-phosphonoacetohydroxamate complex at 2.4-A resolution. Biochemistry, 33, 6295-6300.

17. Larsen, T. M., Wedekind, J. E., Rayment, I. \& Reed, G. H. (1996). A carboxylate oxygen of the substrate bridges the magnesium ions at the active site of enolase: structure of the yeast enzyme complexed with the equilibrium mixture of 2-phosphoglycerate and phosphoenolpyruvate at $1.8 \AA$ resolution. Biochemistry, 35, 4349-4358.

18. Zhang, E., Brewer, J. M., Minor, W., Carreira, L. A. \& Lebioda, L. (1997). Mechanism of enolase: the crystal structure of asymmetric dimer enolase- 2phospho-D-glycerate/enolase-phosphoenolpyruvate at $2.0 \AA$ resolution. Biochemistry, 36, 12526-12534.

19. Grishin, N. V. \& Phillips, M. A. (1994). The subunit interfaces of oligomeric enzymes are conserved to a similar extent to the overall protein sequences. Protein Sci. 3, 2455-2458.

20. Dinovo, E. C. \& Boyer, P. D. (1971). Isotopic probes of the enolase reaction mechanism. J. Biol. Chem. 246, 4586-4593.

21. Anderson, S. R., Anderson, V. E. \& Knowles, J. R. (1994). Primary and secondary kinetic isotope effects as probes of the mechanism of yeast enolase. Biochemistry, 33, 10545-10555.

22. Cohn, M., Pearson, J. E., O'Connell, E. L. \& Rose, I. A. (1970). Nuclear magnetic resonance assignment of the vinyl hydrogens of phosphoenolpyruvate. Stereochemistry of the enolase reaction. J. Am. Chem. Soc. 92, 4095-4098.

23. Reed, G. H., Poyner, R. R., Larsen, T. M., Wedekind, J. E. \& Rayment, I. (1996). Structural and mechanistic studies of enolase. Curr. Opin. Struct. Biol. 6, 736743.

24. Faller, L. D., Baroudy, B. M., Johnson, A. M. \& Ewall, R. X. (1977). Magnesium ion requirements for yeast enolase activity. Biochemistry, 16, 3864-3869.

25. Hanlon, D. P. \& Westhead, E. W. (1969). Equilibrium measurements of the interaction of yeast enolase with activating metal ions. Biochemistry, 8, 4247-4255.

26. Brewer, J. M. (1971). The increase of yeast enolase fluorescence produced by substrates and competitive inhibitors in the presence of excess $\mathrm{Mg}^{2+}$. Biochim. Biophys. Acta, 250, 251-257.

27. Stec, B. \& Lebioda, L. (1990). Refined structure of yeast apo-enolase at $2.25 \AA$ resolution. J. Mol. Biol. 211, 235-248.

28. Holleman, W. H. (1973). The use of absorption optics to measure dissociation of yeast enolase into enzymatically active monomers. Biochim. Biophys. Acta, 327, 176-185.
29. Jones, S. \& Thornton, J. M. (1996). Principles of protein-protein interactions. Proc. Natl Acad. Sci. USA, 93, 13-20.

30. Janin, J., Miller, S. \& Chothia, C. (1988). Surface, subunit interfaces and interior of oligomeric proteins. J. Mol. Biol. 204, 155-164.

31. Mitchell, P., Petfalski, E., Shevchenko, A., Mann, M. \& Tollervey, D. (1997). The exosome: a conserved eukaryotic RNA processing complex containing multiple $3^{\prime} \rightarrow 5^{\prime}$ exoribonucleases. Cell, 91, 457-466.

32. Mitchell, P. \& Tollervey, D. (2000). Musing on the structural organization of the exosome complex. Nature Struct. Biol. 7, 843-846.

33. Coburn, G. A. \& Mackie, G. A. (1999). Degradation of mRNA in Escherichia coli: an old problem with some new twists. Prog. Nucl. Acid. Res. Mol. Biol. 62, 55-108.

34. Gill, S. C. \& von Hippel, P. H. (1989). Calculation of protein extinction coefficients from amino acid sequence data (published erratum appears in Anal. Biochem. (1990);189:283). Anal. Biochem. 182, 319-326.

35. Spring, T. G. \& Wold, F. (1975). Enolase from Escherichia coli. Methods Enzymol. 42, 323-329.

36. Otwinowski, Z. \& Minor, W. (1997). Processing of X-ray diffraction data collected in oscillation mode. Methods Enzymol. 276, 307-325.

37. Navaza, J. (1994). AMoRe: an automated package for molecular replacement. Acta Crystallog. sect. A, 50, 157-163.

38. Collaborative Computing Project, No. 4. (1994). The CCP4 Suite: programs for protein crystallography. Acta Crystallog. sect. D, 50, 469-471.

39. Brunger, A. T., Adams, P. D., Clore, G. M., DeLano, W. L., Gros, P., Grosse-Kunstleve, R. W. et al. (1998). Crystallography \& NMR system: a new software suite for macromolecular structure determination. Acta Crystallog. sect. D, 54, 905-921.

40. Cowtan, K. \& Main, P. (1998). Miscellaneous algorithms for density modification. Acta Crystallog. sect. $D, \mathbf{5 4}, 487-493$.

41. Laskowski, R. A., MacArthur, M. W., Moss, D. S. \& Thornton, J. M. (1993). PROCHECK: a program to check the stereochemical quality of protein structures. J. Appl. Crystallog. 26, 283-291.

42. Kraulis, P. J. (1991). A program to produce both detailed and schematic plots of protein structures. J. Appl. Crystallog. 24, 946-950.

43. Zhu, Z. Y., Sali, A. \& Blundell, T. L. (1992). A variable gap penalty function and feature weights for protein 3-D structure comparisons. Protein Eng. 5, 43-51.

44. Nicholls, A. \& Honig, B. J. (1991). A rapid finitedifference algorithm, utilizing successive over-relaxation to solve the Poisson-Boltzmann equation. J. Comput. Chem. 12, 435-445. 